\title{
Photonic band-gaps bite the dust
}

Hopes that dielectric materials in which the transmission of certain frequencies would be forbidden seem to have been disappointed by the difficulty of realizing expectation and, now, by calculation.

IF only it were possible to make dielectric materials in which electromagnetic waves cannot propagate at certain frequencies, all kinds of almost-magical things would be possible. For example, it would be possible to embed in such a material atoms or molecules in excited states that would literally be incapable of getting rid of their excess energy by radiation if its frequency were in the forbidden range; the result might be a novel state of matter in which atoms or molecules are forever bound to the photons they would emit if their surroundings would accommodate them.

The goal should not be beyond reach. There are familiar examples of how the propagation of waves with certain frequencies is denied. Waveguides, for example, will not accept or transmit microwaves with a frequency below a cut-off frequency determined by their dimensions. In metals, there are allowed and forbidden energies, proxies for frequencies, at which electron waves can propagate without exponential attenuation. The most relevant analogy is that of the perfectly reflecting mirror constructed from several alternating layers of dielectric with alternately high and low refractive index, each of which is a quarter-wavelength thick. So why not look for a threedimensional dielectric solid in which certain optical frequencies would be forbidden in all directions?

Only a year ago, this esoteric search seemed to have succeeded. But now it has been disappointed by the killjoy calculations of two groups of theoreticians. But the non-existence theorem now offered is not conclusive while, for the rest of us, the brief history of this affair is entrancing.

The story begins with Eli Yablinovitch, at the Navesink Research Center of Bell Communications Research, who drew attention to the potential value of materials in which the transmission of light of certain frequencies would be literally impossible. To the extent that the efficiency of devices as different as solar cells and solid-state lasers are determined by the rate at which electrons and holes recombine (with the emission of radiation) in semiconductors, it would plainly be of some importance if that were naturally suppressed by the structure of the material (Phys. Rev. Lett. 58, 2058; 1987).

The potential of the technique was widely and quickly applauded. That some electronic devices might be made dramatically more efficient pointed to an evi- dently important prize. More surprising was the prediction that the properties of many molecular systems, when embedded in such materials, would be modified radically; disassociation energies of homonuclear diatomic molecules, for example, might be substantially reduced. The explanation is that interatomic interactions entailing the interatomic transfer of even virtual photons in the forbidden ranges of frequency will no longer occur.

But how to design and then construct a dielectric material in which the transmission of photons of certain frequencies is forbidden, whatever the direction in which they travel? Photonic band-gap is the word. Three years ago, Yablinovitch outlined a recipe, a three-dimensional analogue of the perfectly reflecting dielectric sandwich, for constructing such a solid from dielectric materials with sharply different refractive indices. If $\lambda$ is the wavelength of the light whose propagation is to be suppressed, first lay down a layer of one material with optical thickness $\lambda / 4$, etch in that a chequerboard array of square pits with the same dimensions, cover that with a similar layer of the second dielectric in which should be etched a similiar chequerboard pattern of holes interdigitating the first . . . . and then repeat the process indefinitely. The end result is a three-dimensional array of blobs of dielectric material with different refractive indices, arranged with the symmetry of a face-centred cubic lattice.

Yablinovitch himself seems to have spent much of the interval since 1987 trying to turn his recipe into reality. To avoid the cost of turning an epitaxial semiconductor fabricator into an experimental tool, he worked with microwaves, meaning that suitable gap-ridden dielectric solids might be fabricated from bulk pieces of dielectric with different refractive indices (dielectric constants) using precision machine-tools. It seems to have been something of a struggle. Last year, Yablinivitch and T. G. Gmitter reported the outcome of their "tedious cut-andtry", or "empirical Edisonian" approach to the problem of realization (Phys. Rev. Lett 63, 1,950; 1989). "Dozens" of structures were machined out of low-loss dielectric material, some containing as many as 8,000 "atoms", or blobs, material with contrasting dielectric constants. Some "atoms" were dielectric spheres, others were spherical hollows filled with air in a sheet of dielectric material.

Most of the "crystals" made in this way appear not to have inhibited microwave transmission, but Yablinovitch was able to report that, provided that the two refractive indices of the dielectric components stand in the ratio of at least 3.5 to 1 , a photonic band-gap does indeed appear. The most successful of his crystals consisted of spheres filled with air machined into a proprietary dielectric material with a refractive index of 3.5 in the frequency range between 1 and $20 \mathrm{GHz}$. Only 14 per cent of the crystal volume was made of dielectric, so that the structure was more closely packed than expected for a facecentred close-packed structure.

In the most successful structure, there seemed to be a band-gap, the width of which seems to have been dependent on the direction of propagation and to be a few per cent of the central frequency whose transmission is inhibited. One evident disappointment was that the contrast of refractive indices required for inhibition turned out to be much greater than expected; another is that there seemed to be no simple relationship between the structure of the composite dielectric crystals and the frequencies at which the band-gaps appear.

This is where the killjoys come in. Those concerned are K. M. Leung and Y. F. Liu from the Brooklyn Polytechnic University, New York, and Ze Zhang and Sashi Satpathi from the University of Missouri at Columbia (Phys. Rev. Lett. 65, 2646 and 2650 respectively; 1990). Each has carried through a calculation of the behaviour expected of a two-component dielectric lattice when full account is taken of the vector character of electromagnetic radiation. It is simply a matter of eliminating the magnetic fields from Maxwell's equations and of treating separately the propagation of plane waves with various directions of travel. There emerges an equation in the form of a determinant of infinite dimension that can be solved numerically by truncation.

What emerges (most clearly from the work of Zhang and Satpathy) is that true band-gaps cannot appear, but that there are circumstances in which the density of transmission states as a function of frequency is not a smoothly varying function, but rather one in which there are so few transmission states in a certain frequency range that the region concerned can be called a "pseudo-gap". On past form, Yablimovitch will no doubt set out to build just such a microwave crystal. That is as it should be.

John Maddox 